\title{
Des livres pour les écoles du peuple ? Économie et pratiques du texte scolaire en Champagne au XVIIIe siècle
}

The economics and practices of school texts in 18th century Champagne Fibeln für die Volksschule? Ökonomie und Praxis des Schultextes in der Champagne im 18. Jahrhundert ¿Libros para las escuelas del pueblo? Economía y prácticas del texto escolar en la región de Champagne en el siglo XVIII

\section{Emmanuelle Chapron}

\section{(2) OpenEdition}

\section{Journals}

Édition électronique

URL : https://journals.openedition.org/histoire-education/2245

DOI : 10.4000/histoire-education.2245

ISSN : 2102-5452

Éditeur

ENS Éditions

\section{Édition imprimée}

Date de publication : 1 juillet 2010

Pagination : 7-34

ISSN : 0221-6280

\section{Référence électronique}

Emmanuelle Chapron, « Des livres pour les écoles du peuple? Économie et pratiques du texte scolaire en Champagne au XVIIIe siècle », Histoire de l'éducation [En ligne], 127 | 2010, mis en ligne le 01 janvier 2014, consulté le 20 mai 2021. URL : http://journals.openedition.org/histoire-education/2245 ; DOI : https://doi.org/10.4000/histoire-education.2245 
Des livres pour les écoles du peuple?

\title{
Économie et pratiques du texte scolaire en Champagne au XVII' siècle
}

\author{
Emmanuelle CHAPRON
}

Parler de texte scolaire au XVIII ${ }^{\mathrm{e}}$ siècle est forcément problématique, tant on sait que les enfants qui fréquentaient les petites écoles d'Ancien Régime faisaient feu de tout bois. Entre leurs mains se trouvaient autant d'ouvrages spécifiquement destinés à un usage pédagogique que d'exemplaires de cette vaste production religieuse (heures, psautiers, instructions chrétiennes), mais aussi laïque (almanachs, romans), qui circulait largement entre les milieux sociaux, entre les sexes et entre les générations ${ }^{1}$. Réduire les lectures des enfants des milieux populaires à celles qui se faisaient entre les murs de la classe serait une autre erreur d'appréciation. Cette " école en miettes " multipliait en effet les occasions d'une fréquentation de l'écrit en famille, auprès des compagnons de champ ou d'atelier, au contact de la bibliothèque du curé de la paroisse ou de la balle du colporteur ${ }^{2}$.

Ces précautions prises, parler de " livre scolaire " n'est pas complètement illégitime. En premier lieu parce que ces objets forment déjà, au début de l'époque qui nous intéresse, une catégorie relativement bien identifiée du champ

1 Dominique Julia, “ Livres scolaires et usages pédagogiques (1660-1780) ", in H.-J. Martin, R. Chartier (dir.), Histoire de l'édition française, t. II, Le livre triomphant (1660-1830), Paris, Promodis, 1984, p. 468-497. Plus largement, Alain Choppin, "Le manuel scolaire, une fausse évidence historique ", Histoire de l'éducation, $\mathrm{n}^{\circ}$ 117, janvier-mars 2008, p. 7-56.

2 Marina Roggero, L'Alfabeto conquistato. Apprendere e insegnare nell'Italia tra Sette e Ottocento, Bologne, Il Mulino, 1999. 
de l'édition et de la librairie. Elle est individualisée dans un certain nombre de catalogues de libraires, comme celui de la veuve Oudot au début des années 1720, qui comporte une rubrique "Livres à l'usage des écoles " nourrie par les presses de Rouen et de Troyes. Dans les décennies qui suivent, les catalogues des libraires parisiens Valleyre, Hérissant ou Barrois font figurer ensemble les heures, psautiers, catéchismes, alphabets mêlés de prières et civilités utilisés dans les écoles ${ }^{3}$. Cette catégorie apparait ensuite dans les mémoires des imprimeurs et des libraires, qui comprennent sous l'expression "livres pour les classes ", souvent accolée à celle d'" usages religieux ", des livres aussi élémentaires que les alphabets. Enfin, cette spécialisation se lit dans les objets typographiques eux-mêmes, puisque l'on voit se multiplier au XVIII ${ }^{e}$ siècle les ouvrages religieux dont le titre indique qu'ils sont spécifiquement destinés aux écoles, Demi-psautier à l'usage des écoles ou Heures à l'usage des écoles ${ }^{4}$.

Dans une contribution de référence à l'Histoire de l'édition française, Dominique Julia avait proposé de " définir le livre scolaire par son usage " ${ }^{5}$. De manière complémentaire, et sans prétendre couvrir l'extraordinaire diversité des matériaux utilisés dans les petites écoles d'Ancien Régime, on se propose d'aborder le livre scolaire tel qu'il est défini par les pratiques de la librairie. Le choix de partir de l'économie éditoriale n'est pas de notre part une façon commode de contourner la question des appropriations par le lecteur, toujours ardue à documenter. C'est au contraire un détour qui y ramène, si l'on suit l'hypothèse que la façon dont ces livres sont produits et distribués dit quelque chose de la manière dont ils peuvent être utilisés. Documenter le circuit du livre suppose d'ailleurs de recourir tant aux archives des chambres syndicales des libraires et imprimeurs et de l'administration royale de la Librairie, qu'à celles des institutions scolaires, écoles paroissiales ou maisons des communautés enseignantes. Sans perdre du regard la variété des situations à l'échelle du royaume, l'ampleur de la question nous a amenée à concentrer l'étude sur

3 Catalogue des livres qui se vendent en la Boutique de la Veuve de Nicolas Oudot, Libraire, ruë de la Harpe, vis-à-vis la ruë du Foin, à côté de la ruë des deux Portes, à l'Image de Notre-Dame, à Paris [années 1720], 4 p., in- $8^{\circ}$ [BnF RES Q-979, pièce 2]. On trouve également sous cette rubrique des ouvrages pédagogiques comme l'École paroissiale et les Avis salutaires aux Peres \& Meres sur l'éducation de leurs Enfans. Pour les autres catalogues, voir, par exemple, Claire Lesage, Ėve Netchine, Véronique Sarrazin, Catalogues de libraires 1473-1810, Paris, Bibliothèque nationale de France, 2006, notices 202, 1467-1469, 2928, 2930. Pour le sens des termes anciens, voir le glossaire en fin d'article, p. 32 .

4 Dominique Julia, "Lectures et Contre-Réforme ", in Guglielmo Cavallo, Roger Chartier (dir.), Histoire de la lecture dans le monde occidental, Paris, Seuil, 2001, p. 324. Dominique Julia, " Livres scolaires et usages pédagogiques ", art. cit. 
une généralité, celle de Champagne ${ }^{6}$. Celle-ci se caractérise, en effet, à la fois par l'existence d'un ancien et important tissu de petites écoles, par celle d'un nombre d'ateliers typographiques qui la place en 1777 au second rang derrière Paris pour la densité du nombre d'imprimeurs par habitant, enfin par celle d'une académie, celle de Châlons, qui met au concours, à la fin du siècle, plusieurs questions sur l'éducation ${ }^{7}$.

\section{I - Livre de peu mais livre de tous : statut et enjeux économiques}

Les livres à l'usage des petites écoles, produit typographique de large diffusion, occupent dans la législation et la pratique de la librairie d'Ancien Régime une place voisine de celle des usages religieux, avec lesquels ils se confondent souvent. Depuis la consolidation du régime des privilèges dans les premières années du XVIII ${ }^{\text {e }}$ siècle, ce sont en grande majorité des ouvrages imprimés sous le régime de la " permission simple ", délivrée en général pour trois ans et ne conférant au libraire aucun monopole sur le titre. Le plus souvent, l'autorisation d'imprimer est reconduite "sans nouvel examen ", comme le précise le registre de l'administration de la Librairie à propos de l'Alphabet pour les petits enfants et de la Civilité puérile, soumis en mars 1725 par Pierre Garnier, le libraire troyen, ou à propos des livres " à l'usage des écoles du diocèse d'Amiens " présentés par l'imprimeur soissonnais Courtois le mois suivant". L'exclusion des ouvrages les plus courants du régime du " privilège général ", qui garantit à un libraire le monopole de la production du titre, repose sur des intérêts bien compris : la nécessité d'une production massive et économique

6 Les villes d'imprimerie de la généralité de Champagne relèvent de trois chambres syndicales différentes : Châlons, Bar-sur-Aube, Épernay, Joinville, Sainte-Menehould, Troyes et Vitry-le-François relèvent de la chambre de Châlons ; Reims, Charleville et Sedan de celle de Reims ; Chaumont et Langres, de celle de Dijon. Voir Georges Lepreux, Gallia typographica, Paris, Honoré Champion, t. II, 1911.

7 Sur le tissu scolaire champenois, Dominique Julia, "L'enseignement primaire dans le diocèse de Reims à la fin de l'Ancien Régime ", Annales historiques de la Révolution française, n 199, 1970, p. 233-286, et "Les petites écoles rurales dans le diocèse de Reims dans la seconde moitié du XVII siècle ", Travaux de l'académie nationale de Reims, 1982, t. 161, L'enseignement élémentaire en Champagne (Contribution au centenaire des lois scolaires de la III République), p. 23-48. Sur la démographie éditoriale, Roger Chartier, "L'imprimerie en France à la fin de l'Ancien Régime ", Revue française d'histoire du livre, t. V, 1975, p. 177-190. Sur l'académie de Châlons, Daniel Roche, Le siècle des Lumières en province. Académies et académiciens provinciaux, 1680-1789, Paris-La Haye, Mouton, 1978, 2 vol.

8 Bibliothèque nationale de France [désormais BnF], ms. fr. $21995, \mathrm{f}^{\circ} 53 \mathrm{v}^{\circ}, 4$ mars $1725 ; \mathrm{f}^{\circ} 56 \mathrm{v}^{\circ}$, 8 avril 1725. 
pour satisfaire aux besoins des populations scolaires, d'une part, l'importance de garantir aux imprimeurs et libraires provinciaux un fonds commun d'ouvrages de débit assuré qui constituent la base de leur survie économique, d'autre part. Les registres de la Librairie ne reflètent malgré tout que très partiellement la masse typographique produite pour les écoles. Avant tout, le matériau le plus élémentaire, l'alphabet-syllabaire, toujours accompagné de prières syllabées et non syllabées, n'est soumis qu'à une simple autorisation du juge local lorsqu'il comporte moins de deux feuilles d'impression, ce qui est souvent le cas ${ }^{9}$. Par ailleurs, un certain nombre d'ouvrages sont inclus dans le privilège général conféré aux évêques pour l'impression des usages de leur diocèse, qu'ils délèguent à un imprimeur de leur choix. C'est notamment le cas pour les " catéchismes d'enfans ", auxquels les évêques du quart nord-est du royaume donnent à partir de la fin du XVII ${ }^{\mathrm{e}}$ siècle le statut d'un véritable livre d'école, sous une forme adaptée aux plus jeunes ${ }^{10}$. Enfin, bien des imprimeurs négligent de solliciter une autorisation d'imprimer pour ces productions si élémentaires, ce que réprouvent régulièrement les arrêts du Conseil du roi, comme celui du 22 juin 1723 qui renouvelle les défenses d'imprimer "aucuns livres, même livres d'usages, de classes ", sans en avoir obtenu privilège ou permission du pouvoir ${ }^{11}$. L'aspiration du monde de la librairie à simplifier ces procédures se traduit dans les premières années du XVIII ${ }^{\mathrm{e}}$ siècle par un projet de "permission générale ", qui serait accordée une fois pour toutes aux libraires et imprimeurs du royaume. Il engloberait les "livres de classes, comme alphabets, rudiments, petits auteurs ", en même temps que d'autres types de produits de large circulation (petits ouvrages de dévotion, bibliothèque bleue) ${ }^{12}$. Les grandes lignes en sont précisées par une circulaire du 20 janvier 1706, adressée par les syndic et adjoint de la Communauté des libraires et imprimeurs de Paris à tous les libraires et imprimeurs du royaume :

9 Les imprimeurs troyens produisent de nombreux $\mathrm{ABC}$ d'une feuille ou une feuille et demie d'impression (BnF, ms. fr. 22 126, notamment $\left.\mathrm{f}^{\circ} 103 \mathrm{v}^{\circ}\right)$. Voir également infra.

10 L'expression se trouve dans un mémoire des libraires et imprimeurs de Paris de 1720 (BnF, ms. fr. 22 072, pièce 35). Voir Dominique Julia, "Figures de l'illettré en France à l'époque moderne ", in Béatrice Fraenkel, Illettrismes. Variations historiques et anthropologiques, Paris, Centre Georges Pompidou, 1993, p. 51-80 ; Jean-Robert Armogathe, "Les catéchismes et l'enseignement populaire en France au XVIII ${ }^{e}$ siècle ", in Images du peuple au XVIII siècle, Paris, A. Colin, 1973, et Aux origines du catéchisme paroissial et des manuels diocésains de catéchisme en France (1500-1660), Paris, Desclée, 1989.

11 BnF, ms. fr. 22067, pièce 180.

12 Voir le glossaire proposé en annexe. 
" pour faciliter l'impression de certains livres communs, Sa Grandeur [le chancelier] nous fait espérer une Permission générale, qui servira pour toujours, à condition que dans toutes les éditions qu'on en fera on se conformera au premier exemplaire qui aura été approuvé, paraphé \& déposé à la Chambre de notre Communauté sans y pouvoir faire aucun changement. Ces livres sont de petits ouvrages de dévotion, comme de petites Heures, même des Usages Romains, le Pensez-y-bien, les Pensées Chrétiennes, \& autres semblables ; des livres de classes, comme alphabets, rudiments, petits auteurs ; livrets en papier bleu, \& autres, dont nous sommes chargez de dresser un catalogue, dans lequel seront marquées les éditions auxquelles on sera tenu de se conformer \& qui pourront être indiquez dans un arrêt, pour en permettre généralement l'impression à tous libraires \& imprimeurs ${ }^{13}$.

La lettre s'achève par une invitation à contribuer à la confection de ce catalogue par l'envoi d'ouvrages.

Le projet est, à notre connaissance, resté sans suite. Cet apparent souci de défense et d'exploitation commune d'un domaine considéré comme " public " ne doit d'ailleurs pas tromper : ce marché scolaire se décline plus souvent de manière concurrentielle. Accessibles aux imprimeurs les plus mal outillés (une presse, quelques fontes suffisent), les livrets scolaires sont également pratiqués par les mieux établis. L'enquête de 1701 sur l'état de la librairie du royaume met en évidence la coexistence de plusieurs régimes de production à l'échelle des villes de Champagne. À Troyes, huit des onze imprimeurs annoncent des petits usages pour les enfants (demi ou quarts de psautiers, heures de Notre-Dame) et des ABC de différentes sortes, de une à trois feuilles d'impression. Le dense maillage scolaire de la France du nord-est soutient la viabilité de ces choix redondants, qui constituent à la fois une planche de salut pour les plus petits et une source de revenus non négligeable pour les plus gros. L'inventaire Oudot de 1722 et celui d'Étienne Garnier en 1789 mettent en évidence, pour chacun, 10 à 15000 exemplaires d'abécédaires et de syllabaires qui, si l'on y ajoute les livrets d'instruction religieuse et de piété à l'usage des enfants, représentent au moins un quart du fonds ${ }^{14}$. Dans les autres villes de Champagne, la production scolaire élémentaire semble moins partagée. À Reims, trois des cinq imprimeurs évoquent des travaux scolaires : Barthélemy Multeau l'aîné et Jean Pierard annoncent des " heures pour les Écoles ", tandis que Barthélemy Multeau le jeune se réserve

13 BnF, ms. fr. 22 071, pièce 223.

14 BnF, ms. fr. 22 126. Sur ces inventaires, Henri-Jean Martin, "Culture écrite et culture orale, culture savante et culture populaire dans la France d'Ancien Régime ", Journal des savants, juillet-décembre 1975, p. 225-282, et Jean Hébrard, "Les livres scolaires de la Bibliothèque bleue : archaïsme ou modernité ? ", in Roger Chartier, Hans-Jürgen Lüsebrink (dir.), Colportage et lecture populaire. Imprimés de large circulation en Europe XVI ${ }^{e}-X X^{e}$ siècles, Paris, IMEC Éditions/Éditions de la Maison des sciences de l'homme, 1996, p. 109-136. 
les "feuilles de classe " destinées au collège ${ }^{15}$. À Châlons, deux seulement des neuf imprimeurs et libraires mentionnent une activité d'impression scolaire au sens large, "livres classiques " pour le collège pour Claude Bouchard, "le gros a.b.c. composé de six feuilles en parangon " pour le libraire Claude Briquet. Mais cette coupe chronologique ne rend sans doute pas complètement compte de la production châlonnaise. Le catalogue des livres imprimés par Jacques Seneuze vers 1679 mentionnait plusieurs sortes d'alphabets ("par syllables [sic], grosses lettres "; " par syllables, avec l'Exercice " ; " contenant les sept Psaumes ") et, si l'on n'en retrouve plus trace en 1701 dans son constat d'activité ni dans celui de Pierre, son fils et successeur, la production n'en a sans doute pas été interrompue $^{16}$. Dans les plus petites cités enfin, les livrets scolaires jouxtent les travaux de ville et les petits ouvrages de dévotion : la veuve Guéry, imprimeur à SainteMenehould, annonce des "a.b.c. pour les enfants " en même temps que des Noëls et des Trésors dévots, tandis que le langrois Claude Personne produit des « livres d'écolier " en même temps que de dévotion ${ }^{17}$.

Les manœuvres déployées par les imprimeurs-libraires pour élargir ou conforter leurs débouchés à l'intérieur de l'espace urbain sont de plusieurs types. La première stratégie revient à décliner pour l'usage scolaire un texte sur lequel l'imprimeur détient une permission ou un privilège. La manipulation peut se résumer à ajouter quelques pages de lettres et de syllabes ou des prières à réciter sur le chemin de l'école à un corpus variable de pièces de dévotion et de liturgie, qui servent à exercer les élèves à la lecture et à la pratique religieuse. À Limoges, l'imprimeur Sardine utilise ainsi un privilège obtenu " pour l'impression des heures, intitulés livre de prieres a l'usage de Limoges " en le faisant servir " a differentes sortes d'heures et avec a.b.c. ", ce qui lui vaut la suspension de son privilège ${ }^{18}$. La seconde stratégie consiste à tenter de monopoliser certains segments de la production scolaire par l'obtention de privilèges, par exemple de privilèges locaux, qui protègent de la concurrence à l'intérieur d'une ville. C'est la démarche de l'imprimeur De Liège, qui obtient en 1718 un privilège local (" pour

15 Sur les feuilles classiques, Marie-Madeleine Compère, Marie-Dominique Couzinet et Olivier Pédeflous, "Éléments pour l'histoire d'un genre éditorial. La feuille classique en France aux XVI et XVII siècles ", Histoire de l'éducation, n 124, 2009, p. 27-49.

16 Catalogue des livres imprimés par J. S. Seneuze, Imprimeur \& Libraire ordinaire du Roy, \& de Monseigneur l'Evesque \& Comte de Chaalons, Pair de France [après 1679], 12 p., p. 12.

17 "Nous auvions trouvé sa boutique garnie de plusieurs livres d'escolier et de devotion et notamment ceux par lui declarés dans son memoire " (BnF, ms. fr. 22 126, fo 50$)$. Il produit notamment des rudiments latins.

18 BnF, ms. fr. 22 071, pièce 205, fo518, "Arrêt contradictoire... ", 16 juillet 1703. 
la ville de Sainte-Manehould seulement ") d'une durée de six ans pour trois sortes d'alphabets (Le gros $A B C$, ou la manière d'apprendre à bien lire en peu de tems à la jeunesse Chrétienne \& Catholique, Alphabet ou Instruction du Chrétien, Nouvel Alphabet ou Instruction du Chrétien), empêchant les deux autres libraires de la ville de les reproduire, même par extraits, ou d'en faire venir d'ailleurs ${ }^{19}$. D'autres libraires poussent plus loin la manœuvre en prétendant détenir un privilège général sur des textes qui n'y étaient auparavant pas soumis. Ces tentatives de mainmise suscitent des réactions d'autant plus violentes qu'elles portent sur des tronçons stratégiques de l'équilibre économique des imprimeurs et qu'elles redoublent l'antagonisme Paris-province. À la fin des années 1680, l'imprimeur châlonnais Jacques Seneuze doit ainsi se défendre contre les attaques du libraire parisien Urbain Coustelier, qui l'accuse d'avoir contrefait un Formulaire de Prières pour les élèves des Ursulines sur lequel il dit avoir un privilège. L'ouvrage mis en accusation est un Manuel des Pensionnaires \& Externes des Religieuses imprimé par Seneuze, contenant les dites prières ${ }^{20}$. La chance de Seneuze est de pouvoir compter dans cette affaire sur la protection de l'évêque de Châlons, qui lui a accordé en 1682 la permission d'imprimer l'ouvrage et obtient l'invalidation de la saisie ${ }^{21}$.

La dernière stratégie est celle du commerce de la contrefaçon. On peut en saisir l'importance en 1777, moment où, après la réforme du système des privilèges, le pouvoir offre aux imprimeurs la possibilité de régulariser leur situation en présentant aux inspecteurs les volumes contrefaits de leurs magasins, qui sont alors estampillés. Dans la Lorraine voisine, plusieurs libraires de Nancy et de Neufchâteau détiennent près de 3500 exemplaires contrefaits du Formulaire de prières à l'usage des pensionnaires des religieuses ursulines, dans des éditions étrangères ou prétendument parisiennes ${ }^{22}$. On trouve également dans leurs magasins des exemplaires du célèbre Magasin des enfans de Madame Leprince de Beaumont dans les éditions de Lausanne, Genève ou La Haye,

19 Voir le privilège du $1^{\text {er }}$ septembre 1718 inséré à la fin de l'Instruction de la jeunesse en la piété chrétienne de Charles Gobinet (Sainte-Menehould, De Liège, 1719).

20 BnF, ms. fr. 22 074, pièce 61, requête de Jacques Seneuze au roi et au Conseil, 1690, in- $4^{\circ}, 4$ p.

21 Extrait [...] pour servir en l'instance qui est au Conseil d'entre Messire Louis-Antoine de Noailles, Evêque Comte de Chaalons, partie intervenante avec Jacques Seneuze, son Imprimeur ordinaire. Contre Anne Bonjean veuve de Damien Foucault, Urbain Coutelier, les Dames Ursulines, \& le Syndic des Libraires de Paris, intervenans (s. 1., 1686).

22 BnF, ms. fr. 21 831. À Neufchâteau, le libraire Monnoyer père détient 938 exemplaires contrefaits de l'édition de Paris, L. G. Dehansy, 1770, tandis que son fils en recèle 1805 exemplaires. À Nancy, le libraire Prévôt fait état de 37 exemplaires du Formulaire dans une édition luxembourgeoise, le libraire Barbier de 668 exemplaires dans une édition nancéenne de 1753. 
versions économiques dont les libraires lorrains assurent le débouché pour un milieu social peut-être assez éloigné des "élèves de la première distinction " qui devaient en être l'auditoire initial ${ }^{23}$. Cette diffusion populaire reste cependant malaisée à saisir autrement que par des indices : sous la Révolution, l'enquête de l'an VI (qui inclut une question sur les livres utilisés dans les petites écoles) montre que l'ouvrage de $\mathrm{M}^{\mathrm{me}}$ de Beaumont est employé dans les classes tenues à Sézanne par la brodeuse Godefroy, une ancienne religieuse ; mais il pourrait s'agir d'un volume d'occasion autant que d'une contrefaçon ${ }^{24}$.

\section{II - Distribuer le livre de classe : libraires, merciers, revendeuses et colporteurs}

Quoique de peu de valeur, ces petits ouvrages de débit sûr représentent, en contexte économique déprimé, un refuge pour les imprimeurs, mais également pour tout un milieu de libraires, merciers, revendeurs et colporteurs. Les canaux de distribution de ces livrets scolaires ne diffèrent pas fondamentalement, dans leur capillarité, de ceux du livre de piété décrits par Philippe Martin²5. En ville, les acteurs de ce marché se répartissent suivant une hiérarchie qui va des gros imprimeurs-libraires aux revendeurs et aux colporteurs urbains, en passant par les libraires et les merciers, à la garniture plus restreinte ${ }^{26}$. Les inventaires des premiers mettent en évidence les multiples déclinaisons des produits scolaires destinés aux plus larges couches de la population, à côté des quelques exemplaires destinés aux enfants des milieux favorisés. La boutique de Pierre Briquet, libraire châlonnais dont l'inventaire est réalisé au moment de la mort de sa femme Jeanne Bernard, le 17 avril 1782, renferme ainsi à la fois des livres d'assortiment en quelques unités (Atlas des enfants, Magasin des enfants, Magasin des adolescents, etc.) et des textes scolaires à

23 Ibid. À Nancy, la veuve Leclerc en détient 43 exemplaires dans l'édition de Genève (L. A. Caille, 1773, 2 vol. in- $8^{\circ}$ ) ; Prévost, 11 exemplaires dans l'édition de Lausanne (J. P. Haubach, 1772, in-12) ; Mathieu, marchand libraire, 133 exemplaires d'une édition de Lausanne.

24 Archives départementales de la Marne [désormais AD Marne], 1 L 1244-1245.

25 Philippe Martin, Une religion des livres (1640-1850), Paris, Le Cerf, 2003.

26 Le droit des merciers à vendre des alphabets, almanachs et petits livrets pieux imprimés hors de la ville est une conquête du XVII e siècle, élargie en 1737 à tous les merciers du royaume, mais encore contestée au début du XVIII siècle par les marchands libraires parisiens. À Reims, suivant des dispositions que l'on retrouve presque identiquement ailleurs, les colporteurs urbains sont théoriquement limités à la vente de livrets de moins de douze feuilles d'impression, imprimés à Reims (BnF, ms fr. 22 126, fo 21-33, Arrest et reiglement pour les imprimeurs, libraires, et relieurs de la Ville de Reims, Reims, chez Nicolas Constant, 1626). 
la douzaine certainement sortis de ses presses, civilités, ABC, catéchismes ou règles chrétiennes. Si l'on s'en tient aux alphabets, on en repère d'au moins trois sortes, allant - suivant qu'ils sont dits " gros " ou " petits ", brochés ou en feuilles - de moins de trois deniers à plus de dix deniers pièce (ill. 1$)^{27}$.

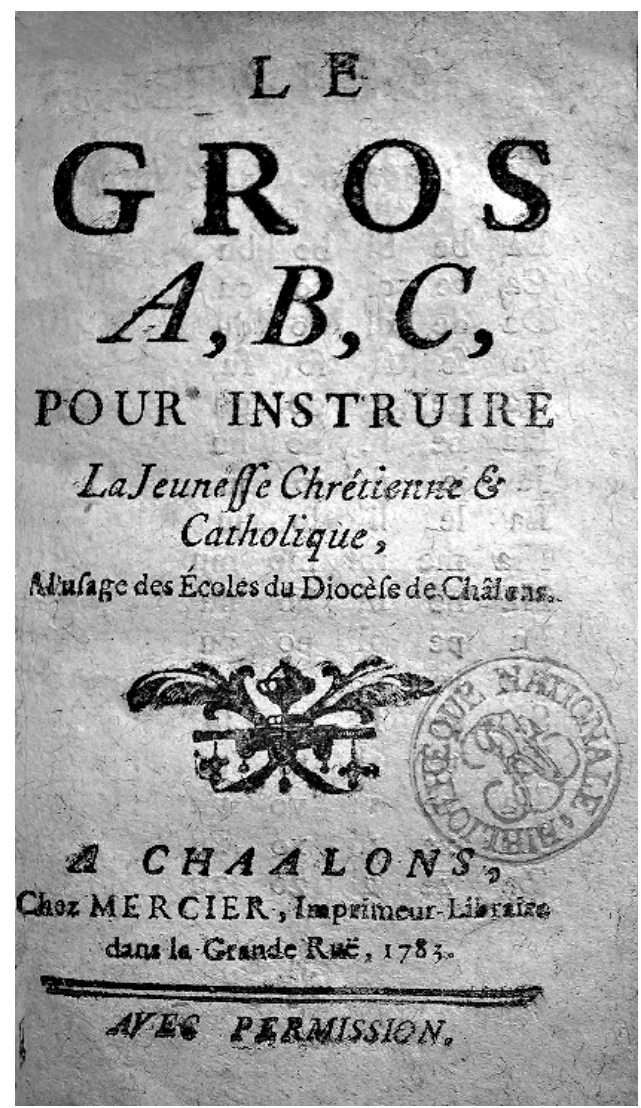

Ill. 1 Le gros A, B, C, pour instruire la Jeunesse Chrétienne \& Catholique, à l'usage des Écoles du Diocèse de Châlons, Chaalons, Mercier, 1783, in-16, 96 p. [BnF RES P-X-474].

Au plus bas de la hiérarchie, figurent les revendeurs, les colporteurs urbains et les petits marchands de foire. On trouve parmi ces derniers des libraires désargentés comme le vieux Gabriel Briden, de Troyes, qui vient au début du siècle vendre sur la foire de Reims les ABC, les psautiers et les almanachs qu'il

27 AD Marne, B 124. Voir également l'inventaire du libraire rémois Barthélemy Multeau (AD Marne, 18 B 292, inventaire des 16-17 janvier 1719) et de Nicolas Pierre Delaître, également libraire à Reims (AD Marne, 18 B 336, 16 novembre 1764). 
imprime chez lui, ou des marchandes de colifichets comme la dame Burien, établie en 1759 sur la foire de Vitry, qui vend "toutes sortes de petites merceries et bijoux à l'usage des enfants " que l'inventaire de ses biens ne prend malheureusement pas la peine de décrire ${ }^{28}$. Autour des écoles gravitent enfin les revendeuses, auxquelles des ordonnances de police défendent de manière répétée, tout au long de la période, d'accepter des livres ou des vêtements en paiement de leurs marchandises ; mais il s'agit plus certainement là d'élèves des collèges ou des pensions privées que des petites écoles ${ }^{29}$. En 1766, lorsque le lieutenant général de police Claude François Bergeat se rend à la chambre syndicale des libraires de Reims pour y examiner les livres saisis depuis plusieurs années, le syndic Barthélemy Multeau et son adjoint lui présentent " des Livres qui ont été saisis par ordre de la Police, sur des tables mises dans le Préau et autres endroits de la Ville, par des gens sans qualité et suspectés de les avoir achetés des Écoliers ". Les écoles rémoises alimentent donc un circuit du livre d'occasion, "classiques et de la Bibliothèque Bleue " ${ }^{30}$.

Loin de rester confinés à l'espace urbain, ces livrets élémentaires alimentent des circuits de diffusion à plus vaste échelle qui, depuis les grosses villes d'imprimerie, fournissent les libraires et revendeurs des plus petites villes, jusqu'aux merciers et colporteurs des campagnes. S'il est possible d'en saisir une partie à travers les correspondances et les bilans comptables post mortem des grands libraires ${ }^{31}$, d'autres documents, rares, permettent d'en percevoir les mécanismes à un niveau intermédiaire. Le livre de compte d'un libraire langrois, Pierre Héron, étudié par Michèle Marsol, montre ainsi comme celui-ci reconstitue chaque année, entre 1754 et 1776, ses stocks d'abécédaires et de livrets pieux auprès de plusieurs imprimeurs : Garnier à Troyes (qui lui livre plus de 500 ABC chaque année), Monnoyer à Neufchâteau (plusieurs cen-

28 Suivant l'enquête de 1701 (BnF, ms. fr. 22 126, f 92), Gabriel Briden, alors âgé de 60 ans, "va alafoire De Reims vendre et debiter des tetres a.b.c. et petits usages (sic) ". AD Marne, B 4391, inventaire des biens de la dame Burien, 21 mai 1759. Sur la librairie de foire, Dominique Margairaz, Foires et marchés dans la France préindustrielle, Paris, Éditions de l'EHESS, 1988.

29 Par exemple, pour Paris, BnF, ms. fr. 22 062, pièce 72 (ordonnance du 10 octobre 1733).

30 Archives municipales de Reims, C 802.

31 Voir le travail effectué par Robert Mandrou à partir de l'inventaire après décès de l'imprimeur troyen Jacques II Oudot (1722) dans De la culture populaire aux $17^{e}$ et $18^{e}$ siècles, préface de Philippe Joutard, Paris, Imago, $3^{\text {e }}$ éd. 1985. 
taines), Collignon à Metz pour des "livres à l'usage des classes "32. La quantité d'alphabets, bien supérieure à ce que peut absorber la population langroise, et la coïncidence des commandes avec la foire de Saint-Mammès, le 18 août, montrent qu'Héron est à l'évidence le fournisseur de plus petits revendeurs, dont le registre ne dit malheureusement rien et qu'il reste difficile de repérer dans l'océan des sources notariées. Outre les colporteurs, que l'on connait par les plaintes des libraires, ses clients pourraient être de ces merciers des gros bourgs situés sur les principaux axes de communication : ailleurs en Champagne, quelques inventaires les montrent plus souvent fournis dans la seconde moitié du XVIII ${ }^{e}$ siècle de livres de piété et de matériel d'écriture. Dans la boutique de Nicolas Sandre, marchand à Fismes, on trouve ainsi en 1784 des plumes, du papier, des écritoires de corne et de bois et des livres de prières ${ }^{33}$.

La concurrence entre professionnels du livre est en réalité moins rude que celle qui les oppose aux particuliers qui se mêlent de commercer des livres scolaires. C'est notamment le cas des maitres et maîtresses, dont les libraires se plaignent beaucoup, d'Abbeville à Avignon, dans les décennies économiquement déprimées du tournant du XVII siècle, mais encore tout au long du XVIII siècle. En 1718, les libraires et imprimeurs d'Abbeville se lamentent que le principal et les régents du collège, ainsi que les maîtres et maîtresses de la ville, "font venir des villes de Rouen \& autres, les livres propres pour leurs écoliers, auxquels ils les vendent à des prix exorbitants, en leur défendant même d'en prendre ailleurs $"^{34}$. Les enjeux économiques de ce détournement apparaissent particulièrement évidents autour des petites écoles des communautés enseignantes, qui rassemblent un nombre important d'élèves et privilégient un petit nombre de titres. Le développement des méthodes de l'enseignement simultané depuis la seconde moitié du XVII ${ }^{\mathrm{e}}$ siècle a, en effet, supposé l'adoption d'un matériel scolaire homogène, auquel encouragent les constitutions de ces congrégations : celles des religieuses de la Congrégation Notre-Dame, présentes dans les principales villes de Champagne, prévoient en 1694 que les maîtresses " tâcheront que [...] toutes les écolières d'une même maitresse aient chacune un même livre en

32 Michèle Marsol, "Un oublié : Pierre Héron "marchand libraire" à Langres en Bassigny, 1756-1776 ", Bulletin de la section d'histoire moderne et contemporaine du Comité des travaux historiques et scientifiques, $\mathrm{n}^{\circ} 11$, Orientations de recherche pour l'histoire du livre, Paris, Bibliothèque nationale, 1978, p. 33-74.

33 AD Marne, B 7164, Inventaire de la boutique de Nicolas Sandre, marchand à Fismes, 6 mars 1784.

34 BnF, ms. fr. 22 067, pièce 176, requête des imprimeurs et libraires de la ville d'Abbeville, s.d., et arrêt du Conseil d'État privé du Roi, 30 avril 1718. 
leur particulier, pour y apprendre et lire toutes ensemble une même leçon " ${ }^{35}$. La difficulté à obtenir des élèves qu'elles se dotent toutes du même livre est en partie résolue par la mise en place d'une distribution par la congrégation ellemême, qui ne s'alimente pas toujours à la source des libraires locaux. En 1757, Joseph Seneuze, imprimeur-libraire à Vitry-le-François, se plaint ainsi au chancelier d'un commerce de livres organisé illégalement par le fils aîné d'un gentilhomme, le sieur d'Arancey ${ }^{36}$. Il l'accuse de faire entrer dans la ville, avec la complicité de la supérieure de l'hôpital, des ballots de livres venus de Paris et, sous couvert de les distribuer ou de les vendre à prix modique aux pauvres, de fournir " les communautés religieuses qui tiennent les écoles dans cette ville ", sans doute la communauté des Dames régentes, ou Nouvelles catholiques. Selon le libraire, " les maitresses des classes les vendent aux écolières, pensionnaires et à toutes personnes qui en veulent acheter, en reçoivent le prix des mains du pauvre et du riche ", le privant ainsi d'une partie de sa clientèle. Pendant quinze ans au moins, Seneuze répète sa plainte, qui se heurte aux intérêts bien compris des élites locales et au subdélégué de l'intendant de Champagne, qui protège ce commerce en arguant des prix trop élevés pratiqués par le libraire ${ }^{37}$.

Le commerce de livres à l'intérieur des communautés religieuses est une réalité bien attestée au XVIII ${ }^{\mathrm{e}}$ siècle. Le registre de comptes des ursulines d'Épernay permet de mesurer l'ampleur de la pratique et, surtout, des bénéfices réalisés (ill. 2) ${ }^{38}$. En 1690, une petite rente de 30 livres est fondée par Nicolas Macquart, directeur des religieuses, pour remplacer la contribution de balayage que les ursulines faisaient jusqu'alors payer à leurs élèves. Cette contribution, puis la rente qui la remplace permettent de payer la reliure des livres des maîtresses, les récompenses promises aux écolières méritantes (chapelets, images pieuses, noisettes, croquants, rubans), du matériel de classe (encre, plumes, canifs pour

35 Les Vraies Constitutions des Religieuses de la Congrégation de Nostre-Dame, faites par le Vénérable serviteur de Dieu Pierre Fourier, Toul, A. Laurent, 1694. Les constitutions prévoient cependant le cas où deux élèves appelées à réciter leur leçon " aprennent dans deux livres divers " : dans ce cas, " elles se mettront toutes deux d'un même côté de la maîtresse, afin que l'une et l'autre puissent voir dans le livre de sa compagne, et remarquer et reprendre ses fautes ". Voir aussi Philippe Martin, "La congrégation de Notre-Dame face au livre ", in Bernard Dompnier, Marie-Hélène Froeschlé-Chopard (dir.), Les religieux et leurs livres à l'époque moderne, Clermont-Ferrand, Presses universitaires Blaise Pascal, 2000, p. 435.

36 AD Marne, C 352.

37 Ibid., le subdélégué de Vitry à l'intendant de Champagne, Vitry, 6 décembre 1757. Une saisie de livres avait déjà été effectuée en 1747, à laquelle Seneuze n'avait pas donné suite, à cause des prières de " personnes notables de Vitry ".

38 AD Marne, 84 H 2/1 : "Livre ou s'escrit l'argent que l'on recoit des contributions des escolieres externes et à quoy il a été employé ". 
tailler les plumes), ainsi que de pourvoir à "l'achat des livres des pauvres qui n'ont pas le moyen d'en avoir, et que nous avons fournies, et pour leur donner de l'encre, des plumes et autres petites nécessités » ${ }^{39}$.

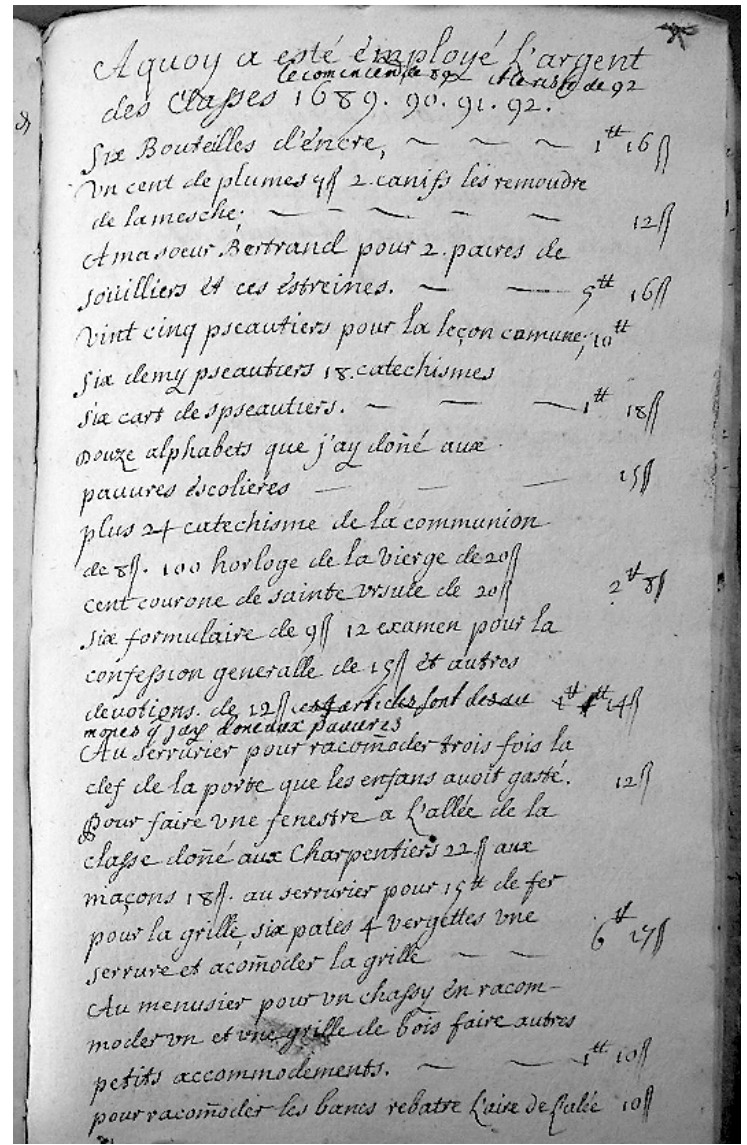

Ill. 2. Extrait du livre comptable des écoles externes des ursulines, compte des dépenses [AD Marne, $84 \mathrm{H} \mathrm{2/1,} \mathrm{p.} \mathrm{8].}$

Les livres achetés se répartissent en trois catégories : les livres " communs de la classe " (p. 14), rangés " dans l'armoire de la maîtresse générale des classes " (p. 25), les livres donnés aux écolières pauvres et les livres vendus aux autres enfants. Les livres destinés aux élèves, psautiers, demi-psautiers et " quarts de psautiers ", catéchismes pour préparer à la première communion, formulaires de prières, examens pour la confession, alphabets, sont achetés en quantités 
moyennes, généralement entre 25 et 30 exemplaires, mais parfois plus : pas moins de dix douzaines de petits catéchismes sont acquis en 1692-1694. Leur revente aux écolières génère un bénéfice relativement important (de 20 à 30 \% du prix d'achat), même si les sommes finales restent modestes : les psautiers achetés 8 sols pièce sont revendus 10 sols, les formulaires de prières à 1 sol 6 deniers sont revendus 2 sols, les petits catéchismes qui reviennent, à la douzaine, à un peu plus de 9 deniers pièce sont revendus 1 sol. Ce bénéfice contribue à l'économie charitable de l'institution scolaire, et notamment aux dons de livres aux écolières les plus pauvres, qui reçoivent des alphabets ou " des livres que l'on nomme trésors dévots".

Ce modeste commerce ne semble pas avoir suscité l'ire des marchands libraires de la ville, au contraire de celui que pratiquent les frères des Écoles chrétiennes. La question des fournitures scolaires des classes lassaliennes est d'autant plus sensible que leurs élèves sont nombreux (environ 35000 dans le royaume à la veille de la Révolution, plus d'un millier dans les cinq écoles de Reims), et que les frères interviennent à plusieurs niveaux dans ce commerce. En amont, le supérieur de l'Institut peut directement traiter avec les imprimeurs, parfois de manière très centralisée, pour un certain nombre de textes de Jean-Baptiste de La Salle pour la publication desquels il a obtenu des permissions simples de l'administration de la Librairie (Syllabaire français, Règles de la civilité et de la bienséance chrétienne, etc. ${ }^{40}$. C'est notamment le cas dans le quart sud-est du royaume, où la maison-mère utilise les services des imprimeurs avignonnais ${ }^{41}$. La question se pose moins dans la moitié nord du royaume, où les libraires obtiennent des permissions simples pour l'impression des volumes lassaliens, notamment la Civilité, dont on connaît au moins six éditions rémoises, cinq édi-

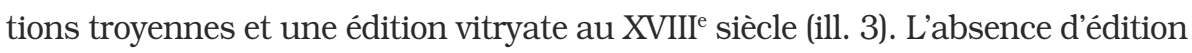
châlonnaise reflète celle des frères (ils ne s'installeront à Châlons qu'au siècle

40 Le 20 février 1726, le frère Timothée, supérieur des frères, obtient ainsi une permission simple pour les "Instructions et exercices de pieté a l'usage des Ecoles chretiennes scavoir un syllabaire francois, un petit exercice de pieté, des instructions et prieres pour la messe, Instructions methodiques pour apprendre a se bien confesser, Catechisme des freres des Ecoles chretiennes par demandes et reponses, les devoirs d'un chretien envers Dieu par discours suivis, Instructions et prieres pour la confession et communion, les regles de la bienseance et de la civilité chretienne, Cantiques spirituels et l'office de la Vierge avec le Psautier de David ", BnF, ms. fr. 21 995, f $73 \mathrm{v}^{\circ}$. Sur ces ouvrages, voir Yves Poutet, "Les livres pédagogiques de Jean-Baptiste de La Salle ", Revue française d'histoire du livre, vol. 49, n²6, 1980, p. 29-67.

41 Voir les documents comptables retrouvés par Yves Poutet, ibid. 
suivant), mais les abondantes productions rémoises alimentent à l'évidence bien au-delà des cinq écoles de la ville ${ }^{42}$.

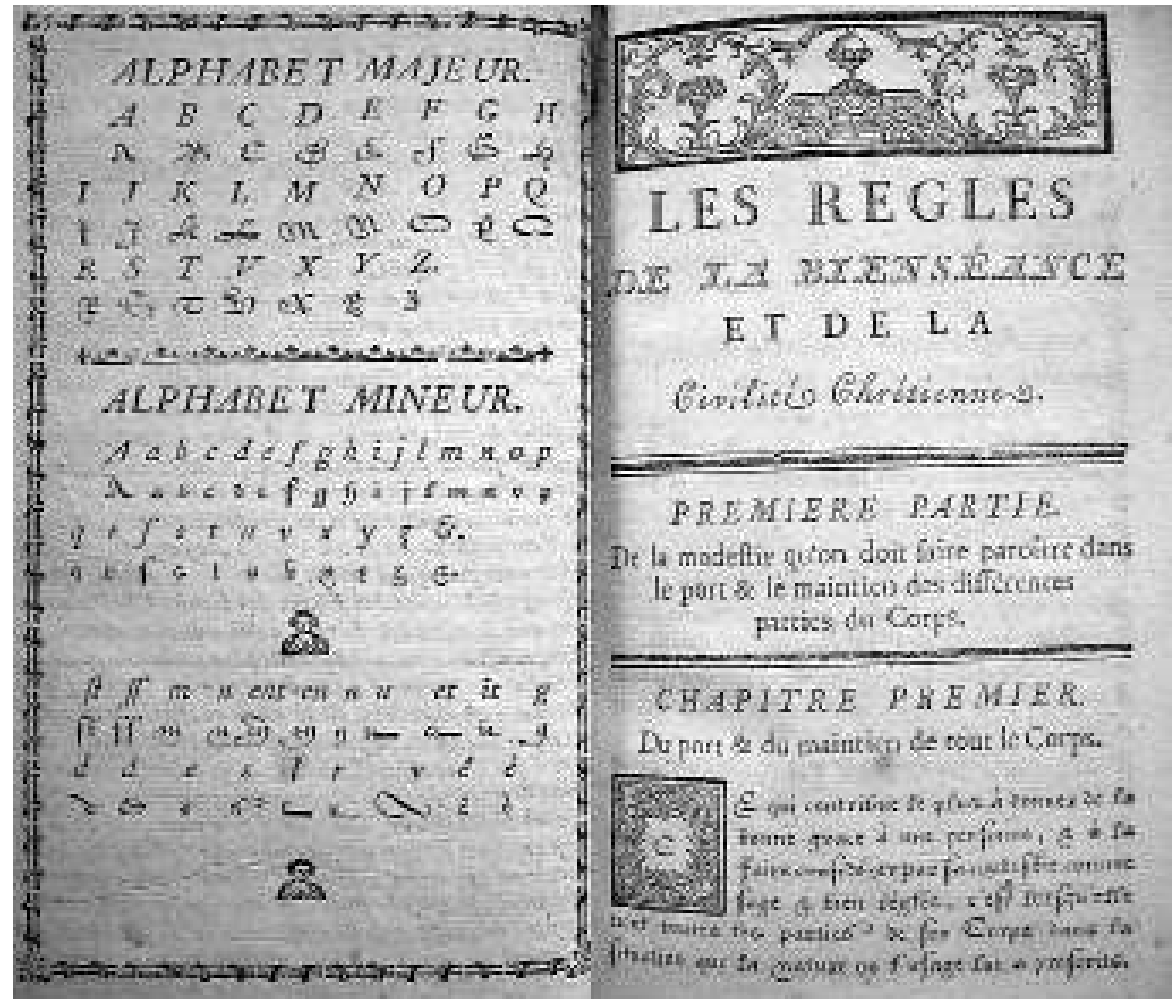

Ill. 3. Les Règles de la Bienséance et de la Civilité chrétienne. Divisées en deux parties. Par Monsieur J. B. de La Salle... Reims, Pierard, 1782.

Le texte en caractères de civilité est précédé d'une table des lettres.

En aval, la fourniture des livres doit garantir la cohérence didactique à l'intérieur des classes. Cette distribution est organisée de manière différente selon les villes, en fonction des termes du contrat d'installation des frères : fourniture par la ville comme à Arras ou à Langres ; par l'évêque, comme à Périgueux sous l'épiscopat de monseigneur de Bouzey ; sur les biens de fondations particulières, comme à Orléans ; ou par les frères eux-mêmes, comme à Nancy. Là où les formes n'en sont pas précisées, les ouvrages peuvent être directement

42 L'édition de Regnault Florentain de 1736 " se vend à Paris chez Gabriel Valleyre ». Le libraire Piérard produit des éditions fréquentes de la Civilité, en 1774, 1779, 1782 (la permission simple porte sur 12000 exemplaires) et 1786 . 
vendus par les frères à leurs élèves, ou donnés aux plus pauvres ${ }^{43}$. Les plaintes contre les frères recouvrent dans ce cas une large gamme de motivations. Celles des libraires, très présentes au début du XVIII siècle, visent le détournement d'une partie du marché de l'édition et de la librairie. C'est le propos de la lettre adressée en 1710 par le syndic des libraires de Reims au contrôleur général des Finances, Nicolas Desmarets, pour faire valoir que " la calamité du temps empêche les particuliers d'acheter des livres [...] et [...] quantité de personnes entreprennent de vendre les seuls livres à présent de débit et nécessaires, comme des heures, usages et livres de dévotion, et prétendent même en avoir la permission, comme les merciers, les Frères et Sœurs des Écoles chrétiennes, qui tirent tous leurs livres du dehors $"^{44}$. Le premier motif de grief est donc que les frères ne se fournissent pas à Reims, mais font venir leurs livres d'ailleurs.

$\mathrm{Au}$ cours du siècle, les plaintes sont plutôt celles des communautés d'habitants et des maîtres concurrencés, qui pointent la contrainte financière et pédagogique exercée par les frères sur leurs élèves. Classiquement, l'attaque se porte sur le prix auquel sont vendus les ouvrages. Lorsque les frères des Écoles chrétiennes s'installent à Avignon au début du XVIII siècle, les maîtres d'école et écrivains publics dénoncent auprès du Conseil de ville la pseudo-gratuité de leurs établissements : " les Frères obligent les enfants à acheter tous les mois leurs livres qu'ils payent trois fois plus cher ; il en est de même pour l'encre, les plumes, le papier, les écritoires, ventes sur lesquelles les Frères font un profit considérable " ${ }^{45}$. La critique n'est que partiellement fondée, car c'est en réalité la maison-mère qui tire profit des ouvrages commandés en quantité importante aux imprimeurs de la cité des Papes, qui sont revendus à prix coûtant par les écoles. En Champagne, la question de la fourniture des livres est agitée en 1772, dans un règlement des écoles produit par le bureau d'administration

43 Annales de l'Institut des Frères des Écoles chrétiennes, t. II, Paris, Procure générale des frères, 1883 , p. 219-220 (Périgueux), p. 491-495 (Arras et Langres). À Nancy, un second contrat fondant une troisième école en 1751 réduit cette obligation aux enfants des trois grandes classes et met en place des fonds pour en doter cinquante enfants des deux dernières classes (ibid., p. 179-180). Georges Rigault, Histoire générale des Frères des écoles chrétiennes, vol. II, Les disciples de Saint Jean Baptiste de La Salle dans la société du XVIII e siècle, 1719-1789, Paris, Plon, 1938, p. 238 (Orléans).

44 Archives nationales, $G^{7} 235$, lettre du syndic des libraires de Reims au contrôleur général, 19 septembre 1710.

45 Michel Feuillas, "Les petites écoles d'Avignon au XVIII" siècle ", Histoire de l'enseignement de 1610 à nos jours. Actes du 95 ${ }^{e}$ Congrès national des sociétés savantes (Reims, 1970). Section d'histoire moderne et contemporaine, t. I, Paris, Bibliothèque nationale, 1974, p. 641-653. 
de la paroisse de Saint-Hilaire de Reims ${ }^{46}$. Le débat porte, encore une fois, sur l'opportunité de sélectionner les élèves des frères, d'en refuser les enfants dont les parents pourraient payer l'écolage et d'en réserver l'accès aux indigents. À l'appui de sa position, le bureau d'administration affirme avoir été informé " que les frères refusent les enfants des pauvres, quoique munis des livres, encre, plumes et papiers à l'usage desdites écoles, sous prétexte qu'ils ne les achètent pas d'eux directement, ce qui est gêner la liberté des pères et mères, d'autant que lesdits frères ne doivent faire aucun commerce de livres, papier, encre et plume ${ }^{47}$. En établissant la liberté des parents de munir leurs enfants de livres achetés " où bon leur semblera ", le bureau protège autant les intérêts de la librairie locale qu'il couvre les pratiques familiales du livre scolaire, faites de transmission et de recyclage plutôt que de dépenses nouvelles. Qu'un article des décisions du Chapitre général de Reims de 1745 impose encore l'emploi " du syllabaire de M. de La Salle dans les écoles " témoigne bien de la difficulté des enseignants à faire adopter partout les instruments lassaliens, même - ou plutôt surtout — au niveau le plus élémentaire, dans des villes saturées d'ABC de toutes sortes ${ }^{48}$.

\section{III - Une économie organisée?}

En dehors des communautés religieuses, la question de l'approvisionnement n'est pas forcément laissée à la "fantaisie des parents " ", et ce d'autant moins que les évêques du XVIII siècle s'emparent de la question des lectures scolaires dans d'autres termes que ceux de leur simple orthodoxie. Certes, les livres sont pour l'essentiel apportés par les enfants. Les inventaires des écoles faits au moment de la Révolution témoignent de cette propriété personnelle des livres de classe. Celui de l'école d'Ay, dirigée par les filles de la communauté de l'EnfantJésus, qui a été dressé le 21 mai 1792 après le départ des sœurs, montre deux salles de classe avec leurs bancs, leurs cartes d'instruction destinées aux plus

46 Le bureau gère les écoles fondées en 1738 par Jean Godinot, docteur en théologie, chanoine et grand-vicaire, et confiées aux frères. Voir les documents publiés dans J. B. Ernest Arnould, Notes et documents sur les établissements d'instruction primaire de la ville de Reims, Reims, P. Regnier, 1848.

47 Ibid., p. 65-68.

48 Georges Rigault, Histoire générale des Frères des écoles chrétiennes, vol. II, Les disciples de Saint Jean Baptiste de La Salle dans la société du XVIII ${ }^{e}$ siècle, op. cit., p. 300.

49 Selon les termes d'un correspondant de l'abbé Grégoire (cité dans Michel de Certeau, Dominique Julia, Jaques Revel, Une politique de la langue, Paris, Gallimard, 1975, p. 239). 
jeunes enfants, les tables à écrire pour les plus grandes, deux tableaux et " une petite armoire aussi [en] bois de chêne à côté servant à y renfermer les livres des enfants, dans lesquels se sont trouvés plusieurs volumes a l'usage des enfants et qui nous a paru leur appartenir " ${ }^{50}$. Bien des indices montrent cependant que dans la seconde moitié du XVIII ${ }^{e}$ siècle, on réfléchit à la manière de fournir les enfants des instruments adéquats.

À partir des années 1750 se multiplient en premier lieu, dans les legs en faveur des écoles, des clauses relatives aux livres et autres fournitures scolaires, à côté des mesures plus traditionnelles concernant la rémunération des maîtres et maîtresses. Des sommes sont destinées par les donateurs à l'achat d'encre, de plumes, de papiers et de livres pour les enfants pauvres : à Matougues en 1750, il est question de "catéchismes et autres livres " pour les "jeunes filles qui ne seront point en état d'en acheter et qui seront assidues aux écoles "; à Varennes en 1757, de " livres d'alphabets, catéchismes " à distribuer " aux enfants des citoyens [...] non aisés " ${ }^{51}$. Les mémoires révolutionnaires feront rétrospectivement état d'autres dotations, comme à Thibie, où une rente du chanoine Briquet au profit des pauvres de la paroisse comprend " dix livres pour fournir des livres, papier, plume et encre aux enfants des deux Écoles " 52 . À moins de disposer de registres de fabrique très précis - ce qui n'est pas le cas ici -, il reste cependant difficile de savoir comment était exactement employé l'argent de ces fondations ${ }^{53}$. Il semble que ces donations aient en tout cas pu donner lieu, dans certains endroits, à la constitution de petites bibliothèques scolaires. Ce phénomène, que Philippe Martin relève en Lorraine dès les premières décennies du XVIII ${ }^{e}$ siècle dans l'entourage des Vatelottes, ne se laisse observer en Champagne que bien plus tardivement, dans les années $1770^{54}$. C'est ce que laissent entendre les dispositions du legs de Claude Maillefert, curé d'Auzécourt, en décembre 1776, qui affecte une partie de la rente, à la gouverne du curé, tant pour

50 AD Marne, E dép. 2483 : inventaire des biens de l'école, 21 mai 1792.

$51 \mathrm{AD}$ Marne, G 94, registre des délibérations de la Chambre ecclésiastique du diocèse de Châlons, $\mathrm{f}^{\circ} 32 \mathrm{v}^{\circ}$, fondation de l'abbé Lemaître ; AD Marne (dépôt de Reims), G 275, extrait du testament de Charles Colin, avocat en Parlement, 20 octobre 1747 et 3 mars 1757.

52 AD Marne, 2 L 216, lettre du président, agent et officiers municipaux de la commune de Thibie aux administrateurs du district de Châlons, s.d.

53 Voir en revanche les comptes retrouvés dans le village d'Auzits par Guy Astoul, Les chemins du savoir en Quercy et Rouergue à l'époque moderne. Alphabétisation et apprentissages culturels, Toulouse, Presses universitaires du Mirail, 1999, p. 198-199.

54 Philippe Martin, Une religion des livres, op. cit., p. 479. 
" entretenir les livres de piété déjà affectés aux écoles dudit Auzécourt, en augmenter le nombre si besoin est, pour l'usage des écoliers et écolières qui sont hors d'état de s'en pourvoir, lesquels livres resteront toujours dans les dites écoles ; que pour leur fournir le papier l'encre les plumes et les catéchismes nécessaires $" 55$.

Cette préoccupation ne semble pas partagée par les communautés d'habitants, s'il faut en croire un exemple unique. En novembre 1783, les habitants du village de Braux-Saint-Remy font parvenir au subdélégué de SainteMenehould une supplique " par laquelle ils proposent, à l'instigation de leur curé, de prendre sur les deniers communs une somme qu'ils ne fixent même pas, pour achat des livres nécessaires pour l'écolage des enfants " ${ }^{56}$. Cette demande originale peut être rapportée aux dispositions prises en amont par l'évêque de Châlons qui, dans les statuts diocésains publiés en 1770, enjoint les maîtres des écoles d'adopter le système de la " leçon générale ", dispensée simultanément à un groupe d'élèves de même niveau et, pour la rendre plus efficace, de faire en sorte que ces élèves " aient tous [...] des livres semblables, et d'une même édition ${ }^{57}$. En vertu de l'arrêt du Conseil du 23 février 1776, qui consacre le principe de la seule compétence de l'intendant ou de son subdélégué à arrêter les comptes communaux et à juger les débats qui s'y rapportent, Mouton rapporte à l'intendant ce projet d'un " genre nouveau de Dépense en fait d'administration des communautés " :

" Je pense qu'il serait en général dangereux de l'admettre, parce que ce serait ouvrir encore aux curés, qui ne s'immiscent déjà que trop dans l'administration des deniers des fabriques, la porte pour gérer encore ceux des communautés, parce que ceux-ci sont spécialement affectés aux réparations des églises, cimetières, presbytères et ponts ; parce qu'enfin ils ne doivent point, suivant l'usage, être employés en achat de livres pour les enfants".

La commune a de nombreuses charges et un budget modeste. Par ailleurs, dans ce très petit village,

" la plupart des habitants sont aisés ; ainsi ils peuvent acheter des livres pour l'écolage de leurs enfants [...] J'ai répondu, de vive voix, au curé qui m'en témoignait, que je croyais que cela ne devait point faire partie de ses sollicitudes pastorales ; je pense que M. l'Intendant me désapprouvera d'autant moins, que quelques habitants m'ont dit que l'achat des livres n'était pas de leur avis, et

55 AD Marne, G 94, $\mathrm{f}^{\circ} 116$, nous soulignons.

56 AD Marne, C 540, lettre de Mouton, subdélégué de Sainte-Menehould, à l'intendant de Champagne, 31 décembre 1783.

57 Abrégé des statuts du diocèse de Chaalons, Châlons, Seneuze [1770], p. 133. 
qu'ils n'avoient signé la délibération que par respect pour leur curé qui la leur avait apportée écrite de sa main " ${ }^{58}$.

L'avis négatif rendu par le subdélégué, puis par l'intendant, manifeste le souci de l'équilibre financier des communautés et le refus de laisser les ecclésiastiques s’immiscer dans leur administration. Il marque également la réticence des autorités royales à favoriser la diffusion des moyens de l'instruction au-delà des couches sociales les plus aisées des campagnes, celles qui peuvent, justement, " acheter des livres pour leurs enfants " ${ }^{59}$. Le faible soutien apporté par les chefs de famille au projet de leur curé ne doit pas faire conclure à un désintérêt pour le matériel scolaire de leurs enfants. Si l'éducation est de plus en plus pensée comme un facteur de promotion sociale, on peut imaginer que les plus riches ne se soucient guère de mettre les deniers communaux au service de l'éducation des enfants pauvres. Il faut par ailleurs rappeler l'économie d'usage de ces livres, transmis à l'intérieur de la fratrie, d'une génération à l'autre, supports d'une continuité des apprentissages élémentaires au sein du groupe familial, que viendrait rompre un achat groupé de livres organisé par le curé ou les autorités villageoises.

\section{IV - Économie d'une réforme scolaire}

Dans les débats des années 1770-1780 consacrés à la question de l'éducation du peuple, la réflexion sur les livres scolaires tient une place secondaire, mais non nulle. Elle découle des attendus d'un projet pédagogique qui soumet les coordonnées de l'éducation populaire (lire, écrire, compter, maîtriser les savoirs techniques et agricoles) aux impératifs de l'utilité économique et de l'ordre social ${ }^{60}$. Elle est le plus souvent abordée à partir des principes qui devraient guider la rédaction d'un livre-somme rassemblant les connaissances adaptées aux enfants du peuple, qui permettrait d'uniformiser les références intellectuelles, morales et religieuses de la population. Cette idée d'un manuel unique court des années 1770 aux premières années de la Révolution. On la retrouve dans les traités consacrés à l'éducation populaire qui paraissent à

58 Ibid., nous soulignons.

59 Sur cette question, Harvey Chisick, The Limits of Reform in the Enlightenment: Attitudes toward the Education of the Lower Classes in Eighteenth-Century France, Princeton, Princeton University Press, 1981.

60 Outre l'ouvrage de Harvey Chisick, voir Alain Choppin, Martine Clinkspoor, Les manuels scolaires en France. 4. Textes officiels (1791-1992), Paris, INRP/Publications de la Sorbonne, 1993. 
partir du milieu des années 1770, dans les dissertations envoyées au concours proposé sur le même sujet en 1779 par l'académie de Châlons, dans les réponses à l'enquête de la Commission intermédiaire de la généralité de Champagne sur l'éducation des enfants pauvres en 1787, et jusque dans les cahiers de doléances ${ }^{61}$. Dans un mémoire de 1787 sur l'éducation des campagnes, l'abbé de Moiremont propose ainsi que l'on fonde deux écoles par bourg, avec des maîtres formés par les frères des Écoles chrétiennes et recrutés sur concours, qui enseigneraient le catéchisme, la lecture, l'écriture, l'arithmétique et des éléments d'agriculture : " Un livre élémentaire sur l'agriculture serait un travail bien digne des lumières de l'Académie de Châlons [...]. La récompense la plus flatteuse pour elle serait sans doute le fruit qui résulterait de son ouvrage distribué dans les paroisses de la campagne " 62 .

Rares sont cependant les projets qui abordent ce livre scolaire comme un objet économique. Parmi eux, on s'arrêtera sur la dissertation qui remporte le concours proposé en 1779 par l'académie de Châlons sur le thème : "Quel serait le meilleur plan pour l'éducation du peuple ? " ${ }^{63}$. Le plan de l'auteur, le parlementaire bordelais Goyon d'Arzac (1740-1800), repose sur un système tripartite d'établissements scolaires (petites, moyennes et grandes écoles), qui correspond à la fois à la hiérarchie urbaine (les seules villes étant dotées de tous les niveaux, limité au premier dans les villages), à une répartition de la population par niveau de richesse (journaliers d'une part, artisans, paysans et marchands d'autre part, répartis en deux sous-groupes) et à une hiérarchie des savoirs (de l'inculcation de rudiments moraux et religieux aux savoirs professionnels). Le livre occupe une part non négligeable des réflexions de l'auteur :

"Autant pour ramener l'éducation du Peuple à l'uniformité désirable, que pour en rendre la pratique plus facile, plus simple et moins coûteuse, nous croyons devoir en fixer la détermination dans des livres à l'usage des écoles populaires, lesquels munis du sceau de l'autorité ecclésiastique et civile et revêtue de la sanction publique, traceraient invariablement soit aux instituteurs, soit aux

61 Sur le livre scolaire dans les cahiers de doléances, voir les documents publiés dans Ernest Allain, La Question d'enseignement en 1789 d'après les Cahiers, Paris, Renouard \& Laurens, 1886.

62 AD Marne, C 2882, Mémoire de l'élection de Sainte-Menehould, adressé par les députés du bureau intermédiaire à la Commission intermédiaire de la généralité de Champagne [1787].

63 BnF, ms. fr. 22 048, fo 51-70, "Essai de Laopédie ou Plan d'éducation pour le peuple. Mémoire adressé à l'Académie des Sciences, Arts et belles-lettres de Châlons-sur-Marne ; accompagné des notes en forme de supplément, et de tableaux figurés qui présentent les détails nécessaires au développement des vues de ce plan. 1781 ", portant les corrections manuscrites du commissionnaire de l'académie, Meunier. Sur l'organisation du concours, Harvey Chisick, The Limits of Reform, op. cit., p. 13-15. 
enfants, soit aux parents eux-mêmes, le genre et la marche de l'éducation, les objets et la forme de l'instruction convenable ${ }^{64}$.

Goyon d'Arzac diffère des autres faiseurs de projets par l'attention portée aux aspects économiques et financiers liés à l'uniformisation des références scolaires. Les ouvrages sont divisés en deux catégories (livres pour les éducateurs et livres pour les élèves) et en trois types (livres d'éducation physique, livres d'éducation morale et religieuse et livres d'éducation didactique). L'instruction des enfants doit se faire sur la base de quatre ouvrages : un catéchisme moral " contenant les devoirs principaux de l'homme dans tous les états de la vie, selon l'ordre de la nature, et considérés relativement à la condition du Peuple ", un catéchisme religieux " renfermant les devoirs essentiels du Chrétien et la Doctrine suffisante pour les gens du peuple ", un Alphabet raisonné à l'usage du Peuple que l'auteur décrit comme fort novateur ("Cet alphabet, d'une espèce nouvelle, simplifiera les principes de la lecture, au moyen d'une exécution typographique et d'une orthographe plus aisée "), enfin des Éléments d'économie rurale, domestique et civile qui réunissent les connaissances "nécessaires à la conduite d'un ménage soit de la ville soit de la campagne ". Ce dernier ouvrage se divise en six parties, dont les deux premières sont des nomenclatures, comme si la formation première des enfants du peuple consistait à les rendre capables de nommer les choses : productions de la nature d'abord, "en commençant par les êtres inanimés, continuant par les êtres inanimés [sic] et finissant par les météores, tous ces êtres rangés par familles ", puis productions de l'art humain " en commençant par les aliments, ensuite par les vêtements, puis par les diverses parties d'une maison, les meubles, les outils les plus usuels et les choses d'un service quotidien ". La troisième partie est un cours d'arithmétique, des règles élémentaires aux opérations ordinaires d'un ménage, tenue de comptes, technique des contrats, baux, achats, ventes, poids et mesures dans leur diversité régionale et leur référence parisienne. Les trois dernières parties rassemblent les connaissances " professionnelles " nécessaires aux mondes de l'agriculture, de l'artisanat et du commerce. À cette bibliothèque scolaire s'ajoutent enfin deux recueils, le premier "d'histoires choisies et d'actions mémorables " tirées des auteurs sacrés et profanes, le second de "cantiques affectueux sur la vie et les mystères de Jésus Christ ". Les livres du maitre consistent en un ouvrage en trois parties intitulé Le conservateur du peuple (il rassemble un manuel des accoucheurs, 
sages-femmes, chirurgiens et orthopédistes, un catéchisme d'orthopédie et un cours de gymnastique populaire) et un Manuel d'éducation populaire qui précise les règles à suivre dans l'enseignement du peuple et la discipline à appliquer ${ }^{65}$.

Ces livres répondent à une exigence, celle du livre unique, somme et quintessence des livres utiles. Ainsi les Éléments sont-ils décrits comme une " espèce d'Encyclopédie élémentaire à l'usage des enfants du Peuple ", comme une monade pédagogique renfermant en un seul traité les moyens d'inculquer aux élèves " les principes et la pratique de la lecture, de l'écriture, de l'arithmétique et du dessin "66. C'est également un outil qui combine l'unicité du support et la possibilité d'une pédagogie socialement différenciée, dans la mesure où Goyon d'Arzac en prévoit des déclinaisons adaptées aux différents niveaux scolaires. Pour les petites écoles dont les élèves ne sont pas destinés à apprendre à lire, des résumés de chaque ouvrage suffiront. Les Éléments peuvent également être disjoints en fonction du public : les six parties seront enseignées dans les grandes écoles, mais seulement les quatre premières parties, " et sans figures afin d'économiser ", doivent suffire aux écoles moyennes, tandis que les petites se contenteront "d'un abrégé succinct mais méthodique de ces quatre parties rédigées en forme de catéchisme économique ". Cette déclinaison se traduit par une gamme de prix : l'alphabet est estimé 15 sols, les catéchismes à 1 livre dans leur version simplifiée mais à 1 livre 5 sols ou 1 livre 10 sols dans leur version complète. Les Éléments coûtent 2 livres en version abrégée, 3 livres dans la version développée sans gravures et 5 livres dans la version complète.

L'auteur résout enfin sans tergiverser plusieurs des questions qui arrêteront quelques années plus tard les assemblées révolutionnaires : qui doit rédiger les ouvrages ? comment les imprimer ? qui doit les financer ${ }^{67}$ Goyon d'Arzac se propose de fournir lui-même les ouvrages : "Quelques-uns sont déjà faits. Les autres ne seraient pas extrêmement difficiles à faire, vu la quantité d'excellents ouvrages qu'on a sur les différents sujets dont il est question. Il ne faut pour cela que de la méthode, de la précision et du zèle. L'auteur de ce Mémoire, s'il est agréé, se chargera volontiers de joindre successivement à son Plan d'éducation les divers accessoires qu'il annonce, trop bien payé, s'il pense contribuer au

65 Ibid., p. 14-15.

66 L'auteur explique en note que les titres devront servir d'exemple pour apprendre à écrire aux enfants, que les définitions et les descriptions des objets pourront être le support d'exercices de lecture, que les renvois chiffrés seront une introduction à la numération, qu'enfin les figures leur fourniront des modèles à recopier à la plume ou au crayon.

Alain Choppin, Martine Clinkspoor, Les manuels scolaires en France, op. cit. 
bonheur de ses semblables !". Il propose que les ouvrages soient imprimés aux frais de l'État dans les chefs-lieux des nouvelles circonscriptions scolaires (les Bureaux provinciaux d'éducation populaire), par des imprimeurs attitrés afin de limiter les transferts de fonds et de préserver l'économie locale de la librairie. Mais les livres resteront à la charge des parents, qui devront se les procurer auprès des bureaux municipaux.

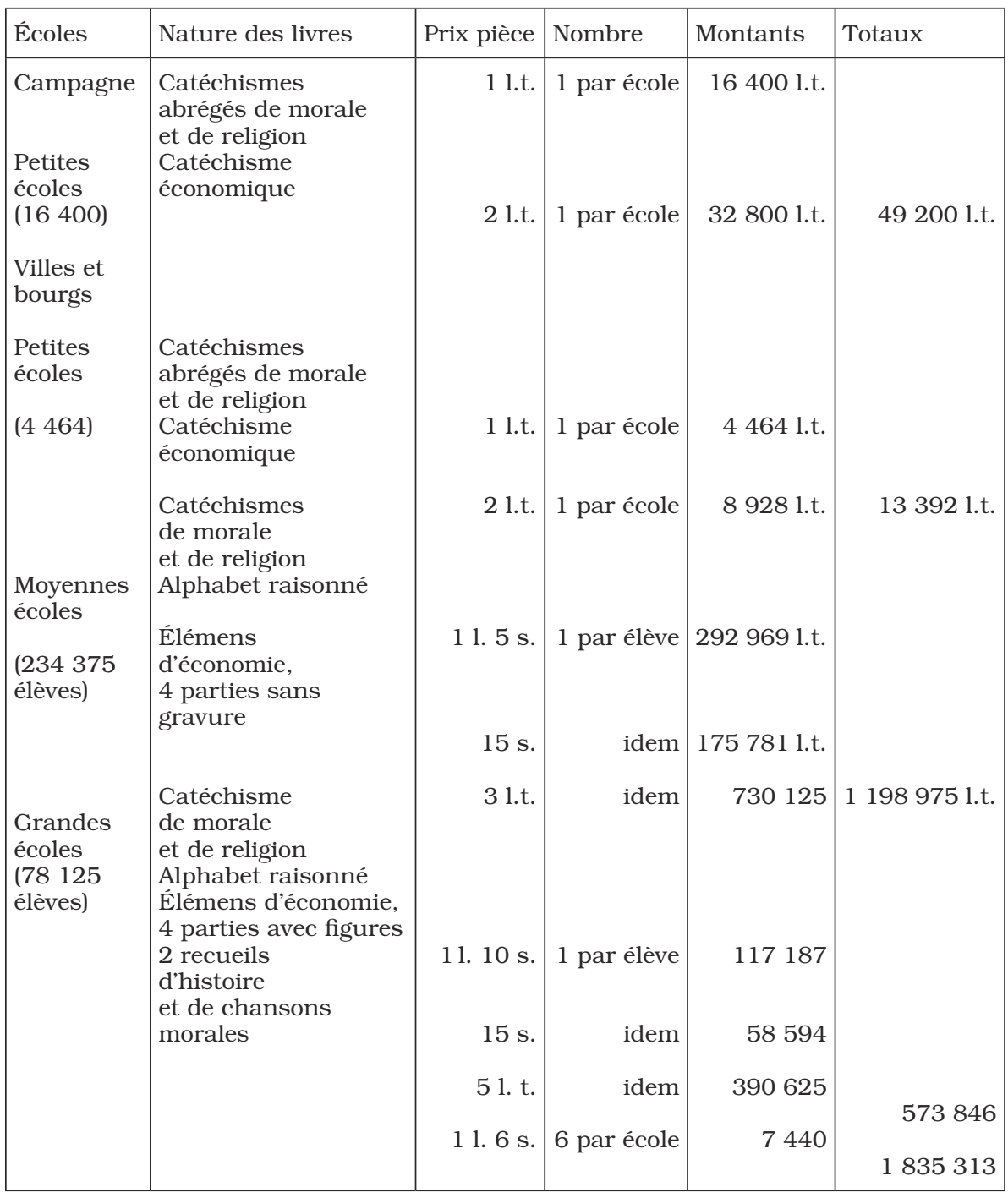

Tableau 1. «État détaillé de la qualité du prix et de la destination des Livres élémentaires ", dans Goyon d'Arzac, Essai de Laopédie..., 1781 (BNF, ms. fr. 22 048, f ${ }^{\circ} 67 v^{\circ}$ ). 
Le "Tableau général de répartition, évaluation \& pour servir à l'intelligence de ce système d'éducation pour le peuple " présente un bilan comptable du projet, qui évalue à près de deux millions de livres la valeur des ouvrages nécessaires à la population scolaire (voir Tableau 1).

Le tableau dressé par Goyon d'Arzac exprime les aspirations d'un certain nombre de réformateurs à asseoir le contrôle de l'État, non seulement sur les contenus pédagogiques ou la réglementation générale de la librairie, mais également sur les formes précises de la production et de la distribution des ouvrages scolaires. Tout un savoir économique et démographique, progressivement mis en ordre et mathématisé dans les dernières décennies de l'Ancien Régime, se trouve exceptionnellement appliqué à la question du marché du livre, dans le cadre des réflexions sur la réforme scolaire ${ }^{68}$. Ce projet d'économie dirigée frappe surtout par son idéalisme, son rejet ou sa profonde méconnaissance des réalités éditoriales et pédagogiques de l'Ancien Régime. Ce sont celles d'un monde du livre attentif à toutes les formes de confiscation d'un secteur qui forme "l'âme des fonds de librairie "69 et est plutôt enclin à déréguler la production scolaire. Ce sont surtout celles d'une population presque constamment réticente à l'adoption d'un matériel de lecture uniforme, alors même que l'enseignement simultané développé depuis le milieu du XVII ${ }^{e}$ siècle dans les écoles charitables parisiennes, dans celles des ordres enseignants, mais également dans les petites écoles paroissiales du XVIII ${ }^{\mathrm{e}}$ siècle, pousse à l'adoption d'un livre unique. Dans ces réticences se mêlent des motifs variés, du refus de consacrer quelques sous à un achat jugé superflu à la permanence d'une représentation de la relation pédagogique sur le mode individuel, en passant par l'attachement à des outils éprouvés, transmis, familiers.

Emmanuelle CHAPRON Université de Provence, Maison méditerranéenne des sciences de l'homme (UMR Telemme) chapron@mmsh.univ-aix.fr

68 Éric Brian, La mesure de l'État. Administrateurs et géomètres au XVIII siècle, Paris, Albin Michel, 1994 ; Jean-Claude Perrot, "L'économie politique et ses livres ", in Henri-Jean Martin, Roger Chartier (dir.), Histoire de l'édition française, t. II, Le livre triomphant (1660-1830), op. cit., p. 298-326.

69 La formule apparait dans un "Mémoire pour les libraires et imprimeurs de Paris, opposans à l'enregistrement d'un Privilege General surpris par M. Cossin recteur de l'université ", 1720 (BnF, ms. fr. 22 072, pièce 35, p. 10). 


\section{Glossaire}

Alphabets : petits ouvrages rassemblant les premiers éléments de l'alphabétisation et les textes liturgiques fondamentaux. D'une structure relativement régulière (même s'ils portent la trace de débats pédagogiques importants), ils comprennent en général : une série d'alphabets proprement dits (majuscules, minuscules, ligatures) en différents corps de caractères (romains, italiques) ; des tables syllabiques organisées par ordre alphabétique et présentant des combinaisons de plus en plus complexes (syllabes de deux, trois et quatre lettres) ; les prières élémentaires (Pater, Ave Maria, Credo), dont les premières singularisent parfois la syllabation par des espacements ou des tirets. D'autres textes liturgiques complètent le volume, au point que l'alphabet proprement dit n'y occupe parfois qu'une place minime. Dans la France de l'Est, ils prennent souvent le titre d'Instructions chrétiennes ou du chrétien ou, comme ailleurs, de croisette ou Croix de par Dieu.

Bibliothèque bleue : ensemble de textes de différents genres (littéraires, religieux, médicaux, etc.) retravaillés et imprimés sous une couverture de papier bleu, que les forts tirages, la pagination limitée, le prix modique et la diffusion par colportage rendent accessible à un très large public rural et urbain. La formule est consacrée par les éditeurs troyens (Oudot, Garnier) dès le début du XVII ${ }^{\mathrm{e}}$ siècle, et adaptée dans d'autres centres éditoriaux (Rouen). À côté des almanachs, des romans, des livres pratiques et de dévotion, on trouve des ouvrages didactiques destinés aux écoliers.

Catéchismes : à partir de la fin du XVII ${ }^{\text {e }}$ siècle, alors que les catéchismes s'uniformisent à l'échelle des diocèses, des adaptations élémentaires en sont composées à l'usage des enfants. Contrairement au "grand ", le " petit catéchisme " ne comprend que les "plus simples éléments de la religion chrétienne " présentés sous forme de questions et réponses (Catéchisme de Reims).

Civilités : imprimées dans le caractère dit "de civilité " inventé au milieu du $\mathrm{XVI}^{\mathrm{e}}$ siècle par l'imprimeur lyonnais Robert Granjon, les civilités présentent les devoirs des enfants envers Dieu et leurs parents, ainsi que des règles de conduite en société. Les volumes intitulés Civilité honnête ou puérile sont souvent des volumes composites, incluant des alphabets et des règles d'arithmétique. Les modèles les plus célèbres en sont l'adaptation du texte d'Érasme par Mathurin Cordier, puis les Règles de la bienséance et de la civilité chrétienne de Jean-Baptiste de La Salle (1 ${ }^{\text {re }}$ éd. 1703), qui en modernisent et en simplifient le propos. Les libraires mettent rapidement en vente des éditions en caractères usuels pour toucher un plus large public.

Feuilles de classe ou Feuilles classiques : présentation éditoriale de textes classiques, latins et grecs, en format in $-4^{\circ}$, d'impression généralement assez grossière, qui ménage de larges espaces marginaux et interlinéaires pour permettre aux professeurs et aux élèves d'y porter leurs notes manuscrites. 
Heures : ouvrage de dévotion polymorphe rassemblant, autour de l'office de la Vierge, des prières et exercices de piété, ainsi que des pièces liturgiques. Les heures pour les enfants comprennent généralement des prières à réciter avant et après l'école.

Livres classiques : on désigne sous cette expression principalement les auteurs latins, grammaires et rudiments (voir ce mot) utilisés dans les collèges d'Ancien Régime.

Livres en papier bleu : voir Bibliothèque bleue.

Pensez-y bien : titre fréquemment adopté par les " arts de mourir ", produit éditorial typique de la réforme catholique.

Pensées chrétiennes : type d'ouvrage de dévotion

Petits auteurs : auteurs enseignés dans les petites classes des collèges (Cicéron, Virgile, Horace, etc.).

Psautier : à l'époque moderne, le terme désigne un genre éditorial polymorphe contenant des pièces de dévotion privée (prières pour différents moments de la journée) et des pièces liturgiques (office du dimanche, vêpres et complies, calendrier et litanies des saints etc.). Les psaumes n'y sont pas présentés dans l'ordre biblique, mais en suivant la structure de l'office. Il est décliné en formes plus brèves appelées " demipsautier" et " quart de psautier".

Rudiments : premiers éléments de la grammaire latine enseignée dans les collèges ou dans les petites régences latines. Le terme reprend l'intitulé du premier volume de la grammaire du flamand Van Pauteren (Despautère), modèle du genre, produit continûment entre le XVI e et le milieu du XVIII ${ }^{e}$ siècle.

Syllabaire françois : composé par Jean-Baptiste de La Salle pour les Écoles chrétiennes, c'est un livre " rempli de toutes sortes de syllabes et de quelques mots ", qui ne doit servir qu'à épeler. La première édition date, au plus tard, de 1698, mais on n'en a conservé aucun exemplaire imprimé avant la Révolution.

Trésor dévot : recueil de pensées pieuses, imprimé en gros caractères et syllabé, servant aux exercices de lecture ; il est également appelé Petit françois.

Usages : ensemble des livres nécessaires à la vie chrétienne. Sont particulièrement destinés aux enfants les psautiers, heures et catéchismes préparant à la communion. 
